\title{
The Use of L-Arginine in the Management of Pre-Eclampsia and Intrauterine Growth Restriction
}

\author{
Hegde C. V.
}

Published online: 20 April 2012

(C) Federation of Obstetric \& Gynecological Societies of India 2012

This article sets out to discuss the role if any of arginine in pregnancy, especially its use in the management/prevention of intrauterine growth restriction and pre- eclampsia. Nitric oxide is a free radical that plays a role in human physiology in various ways. Its role in obstetrics supposedly to induce smooth muscle relaxation and play a mitigatory role in the above mentioned conditions needs to be reviewed. The main site of production of nitric oxide is nitric oxide synthase in endothelial cells, which uses circulating L-arginine as a substrate. Hence, the local availability of this amino acid may be critical to the endothelial adaptive regulatory mechanisms opposing the vasoconstrictors in pre-eclampsia. L-arginine is considered to be a semi-essential amino acid because under increased demands, endogenous synthesis is not sufficient to fulfill requirements [1]. Defective synthesis of a key vasodilator, nitric oxide, has been demonstrated in patients with pre-eclampsia. Circulating L-arginine is a substrate for nitric oxide synthesis during pregnancy; preliminary data suggest that supplemental L-arginine in the diet may lower the risk of pre-eclampsia during pregnancy by promoting vasodilatation through increased production of nitric oxide.

Arginine may be insufficient in pregnancy [2]. Concentrations of asymmetric dimethyl arginine, a competitive inhibitor of nitric oxide synthase, are raised in women with pre-eclampsia [3]. As also endoglin which impairs the

Hegde C. V. ( $\square)$, Professor

Department of OBS/GYN, T N Medical College, Mumbai, India

e-mail: cvvh29@yahoo.co.in; dr.c.v.hegde@gmail.com activation of nitric oxide synthase mediated by transforming growth factor $\beta$ [4]. Supplemental L-arginine in the diet could provide a source of substrate for nitric oxide synthesis during pregnancy, which could promote vasodilatation. There are however limited studies in the use of L-arginine and other nitric oxide donators for preventing pre-eclampsia, as stated in the Cochrane review by Meher and Duley [5]. Existing literature suggests that L-arginine has direct effects on blood pressure in experimental animal models, normal humans, hypertensive patients, women with pre-eclampsia, and healthy pregnant women $[6,7]$. In pre-eclampsia the role of arginine may be limited after the establishment of the condition, as is mentioned by Staff et al. [8] in a study where no effect of L-arginine was manifest when used in women with pre-eclampsia beyond 28 weeks of gestation. Germain et al. [9] disclosed some initial findings in women at high risk of pre-eclampsia who received L-arginine from week 10 of gestation. In a study in 2005 it was seen that in pre-eclamptic patients prolonged dietary treatment with low dose of L-arginine significantly decreased maternal blood pressure and increased bioavailability of endothelial nitric oxide [10].

Intrauterine growth restriction results due to placental insufficiency which in turn may be due to multiple factors including vascular insufficiency. L-arginine ( $3 \mathrm{~g}$ po daily) administered in intrauterine growth restriction seemed to improve estimated fetal weight in a study by Pace et al. [11]. In the past high doses of intravenous L-arginine was used to stimulate growth hormone secretion (Merimee et al.) [12]. However according to Boger et al. [13], oral 
administration of L-arginine in low doses does not produce this effect. However a study in 2004 demonstrated durable improvement in fetal growth and increase in birth weight in patients with intrauterine growth restriction treated with low doses of L-arginine for 20 days [14]. No patients of pre-eclampsia were included in this study. According to Rytlewski et al. [10] the influence of exogenous L-arginine on fetal body weight and for incidence of intrauterine growth restriction was confounding. Prolonged treatment with L-arginine according to them increased fetal weight only transiently and body weights at birth did not differ significantly between L-arginine and placebo groups [15]. Dera et al. [16] in a study in 2007 which included 69 randomly chosen pregnant women diagnosed with gestational hypertension or whose fetuses were diagnosed with intrauterine growth restriction had 42 women receive $3 \mathrm{~g}$ L-arginine daily as a supplement to standard therapy and 27 women receive placebo as well as routine therapy. While their study demonstrated that L-arginine administration to pregnant women with gestational hypertension and growth restriction may improve fetal condition and neonatal outcome after delivery by prolonging pregnancy, the authors concluded that these benefits required confirmation by a larger, more powered study. In contrast in a study which was a double-blind, multicenter, randomized trial in this condition, L-arginine was seen not to be an effective treatment for severe vascular growth restriction [17].

In India L-arginine is available as a $3 \mathrm{gm}$ oral preparation for oral use and is prescribed mainly for the indications discussed in this article. The use of L-arginine in preeclampsia and intrauterine growth restriction can be seen only as one more intervention in the prevention/management of these conditions. Several existing confounding factors in the treatment of these conditions like the use of antihypertensives, antioxidants, nutrients, diet etc. may make it impossible to ascertain the extent of the role L-arginine may play in the mitigation if any, of these conditions. Selection of patients susceptible to preeclampsia early in pregnancy by multiple criteria not agreeable to all researchers may also prove to be a problem in patient selection. As is noted in several reviews more sustained studies would be required in well selected cases to reach conclusions as to the role of L-arginine in the prevention/management of pre-eclampsia and intrauterine growth restriction. It would be incorrect therefore at this point in time to view L-arginine as panacea for the prevention and management of pre-eclampsia and intrauterine growth restriction.

\section{References}

1. Visek WJ. Arginine needs, physiological state and usual diets: a reevaluation. J Nutr. 1986;116:36-46.

2. Morris N, Eaton BM. Nitric oxide, the endothelium, pregnancy and pre-eclampsia. Br J Obstet Gynaecol. 1996;103:4-15.

3. Savvidou MD, Hingorani AD, Tsikas D, et al. Endothelial dysfunction and raised plasma concentrations of asymmetric dimetylarginine in pregnant women who subsequently develop pre-eclampsia. Lancet. 2003;361:1511-7.

4. Venkatesha S, Toporsian M, Lam C, et al. Soluble endoglin contributes to the pathogenesis of pre-eclampsia. Nat Med. 2006; 12:642-9.

5. Meher S, Duley L. Nitric oxide for preventing pre-eclampsia and its complications. Cochrane Database Syst Rev. 2007;2:CD006490.

6. Hishikawa K, Nakaki T, Tsuda M, et al. Effects of systemic L-arginine administration on hemodynamics and nitric oxide release in man. Jpn Heart J. 1992;33:41-8.

7. Campese VM, Amar M, Anjali C, et al. Effects of L-arginine on systemic and renal haemodynamics in salt-sensitive patients with essential hypertension. J Hum Hypertens. 1997;11:527-32.

8. Staff AC, Berge L, Haugen G, et al. Dietary supplementation with L-arginine or placebo in women with pre-eclampsia. Acta Obstet Gynecol Scand. 2004;83:103-7.

9. Germain AM, Valdez G, et al. Letter to the editor: evidence supporting a beneficial role for long term L-arginine supplementation in high-risk pregnancies. Hypertension. 2004;44:e1.

10. Rytlewski K, Olszanecki R, Korbut R, et al. Effects of prolonged oral supplementation with $\mathrm{L}$-arginine on blood pressure and nitric oxide synthesis in preeclampsia. Eur J Clin Invest. 2005;35:32-7.

11. Pace VD, Chiossi G, Facchinetti F. Clinical use of nitric oxide donors and L-arginine in obstetrics. J Matern Fetal Neonatal Med. 2007;20(8):569-79.

12. Merimee TJ, Rabinowitz D, Riggs L, et al. Plasma growth hormone after arginine infusion. Clinical experiences. New Engl J Med. 1967;276:434-9.

13. Boger RH, Bode-Boger SM. The clinical pharmacology of L-arginine. Annu Rev Pharmacol Toxicol. 2001;41:79-99.

14. Sieroszewski P, Suzin J, Karowicz-Bilinska A. Ultarsound evaluation of intrauterine growth restriction therapy by a nitric oxide donor (L-arginine). J Matern Fetal Neonatal Med. 2004;15:363-6.

15. Rytlewski K, Olszanecki R, Lauterbach R, et al. Basic and clinical. Pharmacol Toxicol. 2006;99:146-52.

16. Dera A, Ropacka M, Kowalska J, et al. Arch perinat med. 2007;13(3):35-9.

17. Winer N, Branger B, Azria A, et al. L-Arginine treatment for severe vascular fetal intrauterine growth restriction: a randomized double-bind controlled trial. Clin Nutr. 2009;28(3):243-9. 